\title{
Natural Language Processing for the Web
}

\author{
Silvia Quarteroni \\ Dipartimento di Elettronica e Informazione - Politecnico di Milano \\ Via Ponzio, 34/5 - 20133 Milan, Italy \\ quarteroni@elet.polimi.it
}

\begin{abstract}
The Web offers a wealth of unstructured textual data that is not readily processable using computational resources both because of its format and owing to the ambiguity of natural language. The Natural Language Processing for the Web tutorial focuses on challenging and interesting aspects dealing with natural language Web applications. The audience is introduced to Natural Language Processing as a discipline in order to acquire a basic knowledge of its different methods, particularly statistical, and evaluation metrics. State-of-the-art applications of natural language research will then be discussed in detail, including information extraction from the social Web and Web crawling with particular focus on question answering systems and natural language data service querying.
\end{abstract}

\section{Summary}

The Web offers huge amounts of unstructured textual data that are not readily processable using computational resources. Indeed, the ambiguity of natural language is the main obstacle to its understanding by computers. However, dialogue with artificial intelligences has been a human goal since the Turing test and the first conversational machines appeared in the 1960s, with ELIZA the Rogerian psychotherapist [3].

Fifty years later, we have designed machines able to win TV game shows such as Jeopardy! by giving more correct answers to complicated questions than the all-time top participants [1]; we are able to search the Web via spoken interfaces using our smartphone thanks to billion-word phonetic models [2] and we can make sense of user-contributed data thanks to tagging and what is called the "social Web".

The Natural Language Processing for the Web tutorial illustrates the fundamental building blocks of Natural Language Processing (NLP), with particular attention to statistical models. Furthermore, state-of-the-art applications such as open-domain Web question answering and natural language data service querying are described in higher detail.

Part I: Introduction to Natural Language Processing. This section of the tutorial provides the audience with the main motivations underlying NLP research, which joins aspects of Information Retrieval, Linguistics and Statistics. 
Motivations and Definitions. We define NLP and its main challenges and opportunities; a brief history of NLP with examples from concrete applications is provided.

Levels of Natural Language Understanding. The syntactic, semantic and pragmatic levels of interpretation of textual documents are explained. We discuss syntactic tasks, e.g. Part-of-Speech tagging, shallow parsing and deep syntactic parsing; semantic tasks, including word sense disambiguation, semantic role labeling and recognizing textual entailment; pragmatic and discourse tasks, including anaphora, ellipsis and co-reference resolution.

Methods. Rule-based and Machine learning approaches to NLP are introduced, along with fundamental algorithms for textual classification and sequence labeling. Generative and discriminative models are discussed, including Naïve Bayes, Hidden Markov Models, Support Vector Machines, Conditional Random Fields.

Evaluation. Quantitative assessment of NLP methods include measuring Precision, Recall, F1-measure, Mean Reciprocal Rank, all of which are discussed.

Part II: Applications of Natural Language Processing. This section of the tutorial outlines a few key application of NLP of particular interest to the Web Engineering community. The technology required to convert a natural language query into an "exact" query suitable for an information retrieval engine or a Web service collection is briefly discussed with examples from ongoing research.

Overview of Natural Language Applications. The most widespread applications of NLP include Information Extraction, Machine Translation, Automatic Summarization, Opinion Mining/Sentiment Analysis, Text categorization, (Spoken) Dialogue Systems.

Question Answering. The aim of a Question Answering (QA) system is to reply to queries in natural language with concise answers - not just relevant documents. We illustrate the main components and phases of a QA system: question processing, document retrieval and answer extraction. Advanced QA techniques such as answer re-ranking, interactive and personalized QA are also discussed.

Querying Data Services. Data services allow users to access structured information via Web APIs; however, their interfaces are generally cumbersome for the end-user. We discuss how such a limitation can be overcome by outlining techniques converting queries in natural language into Web Service "logical" queries.

\section{References}

1. Ferrucci, D.A.: Introduction to this is watson. IBM Journal of Research and Development 56(3.4), 36-45 (2012)

2. Franz, A., Milch, B.: Searching the web by voice. In: Proceedings of the 19th International Conference on Computational Linguistics, vol. 2, pp. 1-5. Association for Computational Linguistics (2002)

3. Weizenbaum, J.: Eliza - a computer program for the study of natural language communication between man and machine. Commun. ACM 9(1), 36-45 (1966) 